\title{
Postprandial plasma concentrations of glycine and taurine conjugated bile acids in healthy subjects
}

\author{
K LINNET \\ From the Department of Clinical Chemistry. Frederiksberg Hospital, Copenhagen. Denmark
}

SUMMARY Fasting and postprandial plasma concentrations of glycine and taurine conjugates of cholic, chenodeoxycholic, and deoxycholic acid were measured by a high pressure liquid chromatography-enzymatic assay in nine healthy subjects. The mean value of each bile acid concentration increased significantly $(2 \cdot 4-4 \cdot 7$ times $)$ in the postprandial period. The total glycine/taurine ratio of 2.5 in the fasting state increased significantly to a maximum value of 3.3 at one to $1 \frac{1}{2}$ hours postprandially and then declined. This shift in glycine/taurine ratio shows, that the relative increase in concentrations of glycine conjugates exceeds the relative increase in concentrations of taurine conjugates in the early postprandial period, and supports the view that there is significant absorption of glycine conjugated bile acids from the proximal small intestine.

Measurements of fasting and postprandial serum concentrations of individual bile acids have so far been performed by radioimmunoassay or gas chromatography. ${ }^{1-3}$ Before analysis by gas chromatography the bile acids are deconjugated. so that both free and conjugated bile acids are measured and no information is obtained regarding the amino acid of the conjugate (glycine or taurine). Radioimmunoassays either do not distinguish at all between glycine and taurine conjugates ${ }^{\text {to }}$ or crossreact to a considerable degree. "An analysis of fasting and postprandial patterns of plasma bile acid conjugates separated according to their conjugation has, therefore. not been performed in healthy subjects: yet the diffeient physicochemical characteristics of glycine and taurine conjugates of bile acids - for example. pKa values - are important for their biological handling. A separation according to the two types of conjugate, therefore, would be interesting when investigating the intestinal handling of bile acids.

Using a new high pressure liquid chromatography (HPLC)-enzymatic system. it has been possible to determine separately glycine and taurine conjugates of tri-and dihydroxy-bile acids in plasma. and this report presents an analysis of the postprandial profiles of these bile acids in healthy subjects.

\footnotetext{
* Address for correspondence: Department of Clinical Chemistrs Frederiksherg Hospital, Ndr. Fasanvej 57 . Copenhagen 20010 F. Denmark. Received for publication 1 June 1982
}

\section{Methods}

SUBJECTS

The study was carried out in nine healthy volunteers, four women and five men, with a mean age of 24 years (interval 17-40 years). The subjects were fasted overnight. and in the morning blood samples were drawn from an intravenous cannula kept open by heparin before and at half-hourly intervals after ingestion of a meal consisting of two egg yolks mixed in 0.21 cream with a spoonful of sugar.

\section{ANALYTICAI. METHOD}

Plasma concentrations of glycine and taurine conjugates of cholic, chenodeoxycholic, and deoxycholic acid were measured. ${ }^{9}$ The bile acids were extracted from $6 \mathrm{ml}$ plasma by passing the plasma diluted $(1+19)$ with Tris- $\mathrm{HCl}$ buffer $(0 \cdot 1$ $\mathrm{mol} / \mathrm{l}$. pH 8.0) through an octadecylsilane column, from which the adsorbed bile acids were eluted by methanol. A HPLC system with a uBondapack $C_{18}$ (Waters) column and a mixture $(65 / 35, v / v)$ of methanol and $0.020 \mathrm{~mol} / \mathrm{KH}_{2} \mathrm{PO}_{4}$ buffer, $\mathrm{pH} 5 \cdot 30$, as a mobile phase at a flow rate of $1.4 \mathrm{ml} / \mathrm{min}$ was used to separate the individual bile acids. These were measured by enzymatic fluorometry with a $3 \alpha$-hydroxysteroid dehydrogenase - diaphorase system with $\mathrm{NAD}^{+}$as cofactor and resazurin as fluorogen (Sterognost $3 \alpha \mathrm{Flu}$. Nycomed). Recoveries ranged from 81 to $96 \%$ and coefficients 
of variations from 5 to $15 \%$. Detection limits (defined as $3 \times \mathrm{SD}$ of a low repetent) were 0.08 $\mathrm{nmol} / \mathrm{ml}$ for glycodeoxycholic acid and from 0.03 to $0.06 \mathrm{nmol} / \mathrm{ml}$ for the other bile acids when $30 \%$ of the plasma extract was injected in the chromatograph; the sensitivity was increased by injecting $45 \%$ of the plasma extract when fasting samples were analysed.

\section{STATISTICS}

The significance of differences between mean values was evaluated by Student's $t$ test (paired).

\section{Results}

The mean values of the fasting plasma bile acid concentrations ranged from $0.07 \mathrm{nmol} / \mathrm{ml}$ (taurodeoxycholic acid) to $0.24 \mathrm{nmol} / \mathrm{ml}$ (glycochenodeoxycholic acid) (Table 1). The postprandial profiles of the bile acids are shown in Figs. 1-3. Each bile acid concentration had already risen significantly at a half hour postprandially $(\mathrm{p}<0 \cdot 01)$. The highest postprandial levels were from $2 \cdot 4$ (taurocholic and taurodeoxycholic acid) to 4.7 times (glycochenodeoxycholic acid) higher than the fasting values. Generally, the glycine conjugates obtained a clearly defined maximum value within the $2 \frac{1}{2}$ hour period studied, whereas the taurine conjugates were still increasing at the end of the period (taurochenodeoxycholic acid), or reached a broad plateau (taurocholic and taurodeoxycholic acid). The difference in postprandial profiles between glycine and taurine conjugated bile acids becomes more pronounced when the profile of the added glycine conjugates is compared with the profile of the combined taurine conjugates (Fig. 4).

The different postprandial plasma concentration patterns of glycine and taurine conjugated bile acids lead to a shift in the glycine/taurine ratios as seen in Fig. 4 and Table 2. The glycine/taurine ratio for the

Table 1 Fasting and postprandial plasma concentrations of conjugated bile acids ( $\mathrm{nmol} / \mathrm{ml}$ )

\begin{tabular}{|c|c|c|c|c|c|}
\hline & \multicolumn{2}{|c|}{ Fasting level } & \multicolumn{3}{|c|}{$\begin{array}{l}\text { Highest } \\
\text { postprandial level }\end{array}$} \\
\hline & Mean & $S E M$ & Mean & $S E M$ & Time $(h)$ \\
\hline Glycocholic acid & $0 \cdot 17$ & $0 \cdot 02$ & 0.59 & $0 \cdot 10$ & 1 \\
\hline Taurocholic acid & $0 \cdot 10$ & $0 \cdot 01$ & $0 \cdot 24$ & $0 \cdot 06$ & 2 \\
\hline $\begin{array}{l}\text { Glycochenodeoxy- } \\
\text { cholic acid }\end{array}$ & $0 \cdot 24$ & $0 \cdot 03$ & $1 \cdot 13$ & $0 \cdot 29$ & 2 \\
\hline $\begin{array}{l}\text { Taurochenodeoxy- } \\
\text { cholic acid }\end{array}$ & 0.09 & $0 \cdot 01$ & $0 \cdot 29$ & $0 \cdot 04$ & $2 \frac{1}{2}$ \\
\hline Glycodeoxycholic acid & $0 \cdot 17$ & 0.02 & 0.51 & 0.07 & $1 \frac{1}{2}$ \\
\hline Taurodeoxycholic acid & $0 \cdot 07$ & 0.006 & $0 \cdot 17$ & 0.03 & $1 \frac{1}{2}-2 \frac{1}{2}$ \\
\hline
\end{tabular}

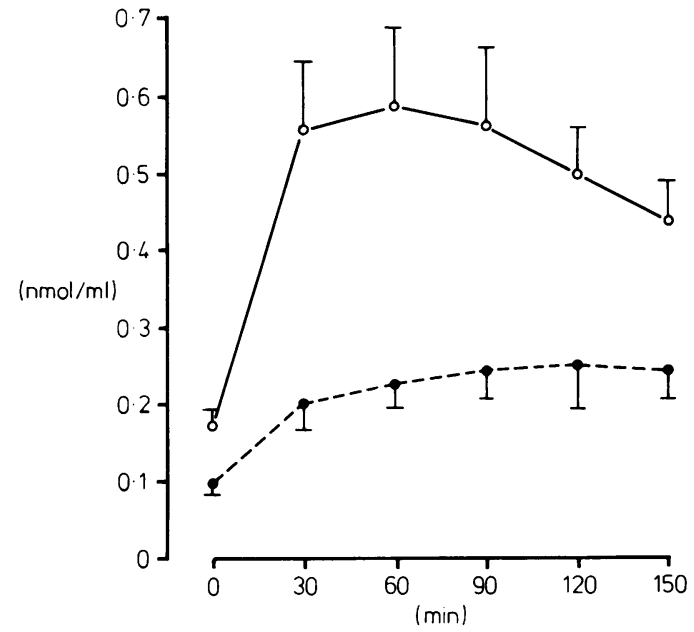

Fig. 1 Postprandial plasma profiles of glycocholic (-) and of taurocholic acid (- - ) (mean and SEM).

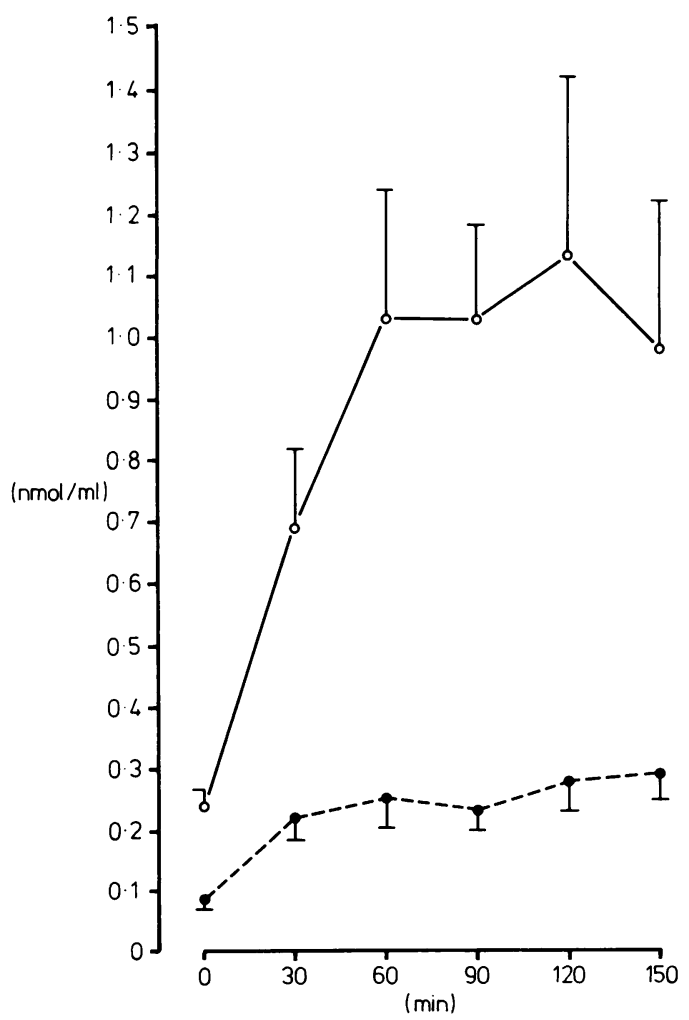

Fig. 2 Postprandial plasma profiles of glycochenodeoxycholic (-) and of taurochenodeoxycholic acid (- - ) (mean and SEM). 


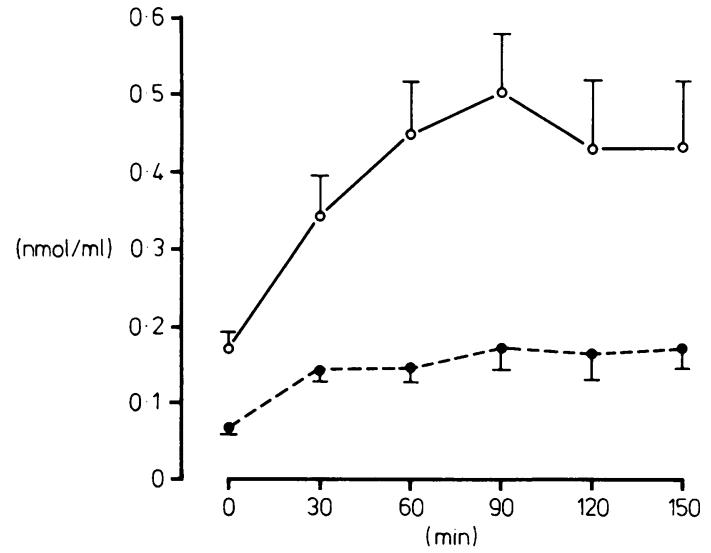

Fig. 3 Postprandial plasma profiles of glycodeoxycholic $(\longrightarrow)$ and of taurodeoxycholic acid $(--$,$) (mean and$ SEM).

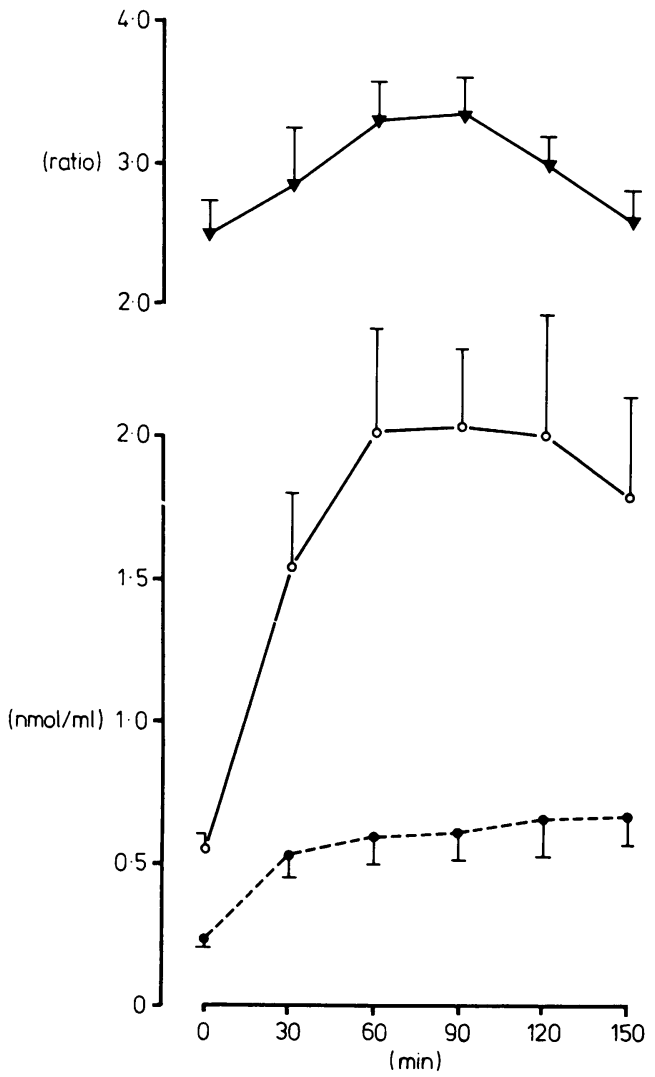

Fig. 4 Top: total glycine/taurine ratio in postprandial period. Bottom: postprandial plasma profiles of sum of glycine conjugates (-) and of the sum of taurine conjugates (- - ) (mean and SEM).
Table 2 Fasting and postprandial glycine/taurine ratios (mean and SEM) of conjugated bile acids in plasma

\begin{tabular}{|c|c|c|c|c|c|c|}
\hline & \multicolumn{6}{|c|}{ Time $(h)$} \\
\hline & 0 & $\frac{1}{2}$ & 1 & $l_{2}^{\frac{1}{2}}$ & 2 & $2 \frac{1}{2}$ \\
\hline \multicolumn{7}{|l|}{ Bile acid } \\
\hline Cholic acid & $\begin{array}{c}1.92 \\
(0.22)\end{array}$ & $\begin{array}{l}3 \cdot() 6^{*} \\
(() \cdot 53)\end{array}$ & $\begin{array}{c}2.83 \\
(0.42)\end{array}$ & $\begin{array}{c}2 \cdot 46 \\
(0 \cdot 36)\end{array}$ & $\begin{array}{c}2.38 \\
(0.25)\end{array}$ & $\begin{array}{r}1.98 \\
(0.19)\end{array}$ \\
\hline $\begin{array}{l}\text { Chenodeoxycholic } \\
\text { acid }\end{array}$ & $\begin{array}{c}3.25 \\
(0.51)\end{array}$ & $\begin{array}{l}3 \cdot(03 \\
(0 \cdot 42)\end{array}$ & $\begin{array}{l}3.95 \\
(() \cdot 35)\end{array}$ & $\begin{array}{l}4.7()^{*} \\
(0.67)\end{array}$ & $\begin{array}{l}3.76 \\
(0 \cdot 42)\end{array}$ & $\begin{array}{r}3.18 \\
(0.36)\end{array}$ \\
\hline Deoxycholic acid & $\begin{array}{c}2.67 \\
(0.26)\end{array}$ & $\begin{array}{c}2.43 \\
(0.35)\end{array}$ & $\begin{array}{c}3.16 \\
(0.42)\end{array}$ & $\begin{array}{c}3.31 \\
(() .35)\end{array}$ & $\begin{array}{c}2 \cdot 68 \\
(0 \cdot 28)\end{array}$ & $\begin{array}{c}2.51 \\
(0.31)\end{array}$ \\
\hline Total & $\begin{array}{c}2.50 \\
(0.24)\end{array}$ & $\begin{array}{c}2.86 \\
(0.40)\end{array}$ & $\begin{array}{c}3.29^{*} \\
(0.29)\end{array}$ & $\begin{array}{c}3.35 \div \\
(0.26)\end{array}$ & $\begin{array}{c}3.00 \\
(0.20)\end{array}$ & $\begin{array}{l}2.60) \\
(0.23)\end{array}$ \\
\hline
\end{tabular}

${ }^{*} \mathrm{p}<0 \cdot(05 . \div \mathrm{p}<0 \cdot(025$ for the difference between value at time 0 .

total bile acids measured increases significantly from a fasting value of 2.5 to maximum value of 3.3 at one to $1 \frac{1}{2}$ hours postprandially, and then declines towards the initial value. The glycine/taurine ratios of all three bile acids increase postprandially, but the changes reach statistical significance only for the primary bile acids.

\section{Discussion}

This study of fasting and postprandial plasma concentrations of glycine and taurine conjugated bile acids in healthy subjects revealed a difference between the profiles of these two types of conjugates. The postprandial increase in the total glycine/taurine ratio to a maximum at one to $1 \frac{1}{2}$ hours shows that, in the early postprandial phase, the relative increase in plasma concentrations of glycine conjugated bile acids exceeds the relative increase of taurine conjugated bile acids. The plasma bile acid level is determined by the balance between intestinal input and hepatic clearance and represents the hepatic 'spill over'. The hepatic assimilation of bile acids under physiological conditions follows the kinetics of a first order reaction with uptake of a constant fraction of the bile acids carried to the liver. ${ }^{10}$ The hepatic fractional uptake of the glycine conjugate of a bile acid is identical with the uptake of the taurine conjugate, and the uptake of conjugated cholic acid is higher than the uptake of a conjugated chenodeoxycholic acid. ${ }^{11}$ Thus, the larger relative postprandial increase in glycine compared with taurine conjugates of the bile acids in the early postprandial period is most likely to depend on a larger intestinal absorption rate of these conjugates.

There is evidence for two types of absorption mechanisms for bile acids in the small intestine: 
passive absorption in both the jejunum and ileum. and active absorption confined to the ileum. ${ }^{12}{ }^{13}$ The passive absorption rate depends on the polarity of the bile acid: dihdroxy-bile acids are absorbed faster than trihydroxy-bile acids, unconjugated bile acids faster than conjugated bile acids, and protonated forms faster than ionised forms. The state of ionisation is of particular importance as the permeability for protonated forms is about 10 times that for ionised forms. ${ }^{12}$ The active transport system in the ileum on the other hand favours absorption of trihydroxy-bile acids over dihydroxy-bile acids and conjugated forms over free forms with only minor differences for tauro- compared with glycoconjugates.

The $\mathrm{pKa}$ values of glycine conjugated bile acids are nearly 5 compared with about 2 for tauroconjugates. A sufficient fraction of glycine conjugates, therefore, should be protonated at least in the most proximal part of the intestine to allow for a non-ionic diffusion across the intestine, whereas this should not be possible for taurine conjugates. The higher relative increases in the early postprandial period of glycine conjugates compared with the taurine conjugates are thus explained by a passive, non-ionic absorption in the proximal intestine of glycine conjugated bile acids, in addition to the active absorption in the ileum of both types of conjugates. Somewhat unexpectedly, glycocholic acid tended to increase more rapidly than the less polar glycochenodeoxycholic acid and glycodeoxycholic acid, and correspondingly the glycine/ taurine ratio of the trihydroxy-bile acid increased earlier than the ratios of the dihydroxy-bile acids. The concentration of glycocholic acid again declined faster than the concentrations of the glycodihydroxy-bile acids, which is in accordance with a higher hepatic clearance of the former. The different absorption patterns of glycine and taurine conjugated bile acids should be borne in mind when, as suggested by some authors, ${ }^{14}$ the postprandial plasma bile acid profile obtained by a radioimmunoassay procedure is used as a test of ileal function.

\section{References}

1 LaRusso NF. Korman MG. Hoffman NE. Hofmann AF. Dynamics of the enterohepatic circulation of bile acids. $N$ Engl J Med 1974; 291: 689-92.

2 Angelin B, Björkhem I. Postprandial serum bile acids in healthy man. (iut 1977; 18: 606-9.

3 Schalm SW, LaRusso NF. Hofmann AF. Hoffman NE. van Berge-Henegouwen GP. Korman MG. Diurnal serum levels of primary conjugated bile acids. (iut 1978: 19: 1006-14.

4 Simmonds WJ. Korman MG. Go VLW. Hofmann AF. Radioimmunoassay of conjugated cholyl bile acids in serum. Gastroenterology 1973; 65: 7(1)5-11.

5 Schalm SW, van Berge-Hengouwen GP. Hofmann AF. Cowen AE. Turcotte J. Radioimmunoassay of bile acids: Development, validation, and preliminary application of an assay for conjugates of chenodeoxycholic acid. Gastroenterology 1977: 73: 285-9().

6 Roda A, Roda E, Aldini R et al. Results with six "Kit" radioimmunoassays for primary bile acids in human serum intercompared. Clin Chem 1980); 26: 1677-82.

7 Demers LM. Hepner G. Radioimmunoassay of bile acids in serum. Clin Chem 1976; 22: 6()2-6.

8 Miller P, Weiss S, Cornell M, Dockery J. Specific ${ }^{125}$ I-Radioimmunoassay for cholylglycine, a bile acid. in serum. Clin Chem 1981; 27: 1698-1703.

9 Linnet K. A high pressure liquid chromatography enzymatic assay for glycine and taurine conjugates of cholic, chenodeoxycholic, and deoxycholic acid in serum. Scand J Clin Lab Invest 1982; 42: 455-6().

10 Ahlherg J, Angelin B, Björkhem I, Einarsson K. Individual bile acids in portal venous and systemic blood serum of fasting man. Gastroenterology 1977: 73: 1377-82.

11 Cowen AE, Korman MG, Hofmann AF. Thomas PJ. Plasma disappearance of radioactivity after intravenous injection of labelled bile acids in man. Gastroenterology 1975; 68: 1567-73.

12 Schiff ER, Small NC. Dietschy JM. Characterisation of the kinetics of the passive and active transport mechanisms for bile acid absorption in the small intestine and colon of the rat. J Clin Invest 1972; 51: 1351-62.

13 Krag E, Phillips SF. Active and passive bile acid absorption in man. J Clin Invest 1974; 53: 1686-94.

14 Balistreri WF, Suchy FJ, Heubi JE. Serum bile acid response to a test meal stimulus: a sensitive test of ileal function. J Pediatr 1980; 96: 582-89. 